\title{
Small is Big: Magic Nanoliter Droplets
}

\author{
Liqiu Wang \\ Department of Mechanical Engineering, The University of Hong Kong \\ Pokfulam Road, Hong Kong \\ Lqwang@hku.hk
}

Droplets of nanoliter and subnanoliter are useful in a wide range of applications, particularly when their size is uniform and controllable. Examples include biochemistry, biomedical engineering, food industry, pharmaceuticals, and material sciences. One example of their many fundamental medical applications is the therapeutic delivery system for delivering sitespecific therapy to targeted organs in the body and as the carriers for newer therapeutic options. The size, the size distribution, the generation rate and the effective manipulation of droplets at a scale of nano, pico, femto and even atto liters are critical in all these applications. We make an overview of microfluidic droplet generation of either passive or active means and report a glass capillary microfluidic system for synthesizing precisely controlled monodisperse multiple emulsions and their applications in engineering materials, liquid-repellent surfaces, nanofluids, thermal-wave fluids, microfibers, embolic particles, artificial blood vessels and colloidosome systems. Our review of passive approaches focuses on the characteristics and mechanisms of breakup modes of droplet generation occurring in microfluidic cross-flow, co-flow, flow-focusing, and step emulsification configurations. The review of active approaches covers the state-of-the-art techniques employing either external forces from electrical, magnetic and centrifugal fields or methods of modifying intrinsic properties of flows or fluids such as velocity, viscosity, interfacial tension, channel wettability, and fluid density, with a focus on their implementations and actuation mechanisms. Also included is the contrast among different approaches of either passive or active nature. 